\title{
Oportunidades educativas y características familiares en Colombia: un análisis por cohortes
}

\author{
Recibido: Junio 15 de 2013 - Aceptado: Mayo 2 de 2014
}

Doi: dx.doi.org/10.12804/rev.econ.rosario.17.01.2014.06

\author{
Carlos Giovanni González Espitia* \\ Universidad Icesi
}

Jhon James Mora Rodríguez ${ }^{\dagger}$

Universidad Icesi

Andrés Felipe Cuadros Meñaca ${ }^{\ddagger}$

University of Arkansas

Resumen

En este artículo se analiza la relación entre las características familiares y el acceso a la educación posobligatoria (media y superior) en Colombia. Para ello, se estima un Modelo Probit ordenado generalizado usando siete cohortes con los datos de la Gran Encuesta Integrada de Hogares (GEIH) para el periodo 2008-2012. Los principales resultados econométricos muestran que características como la educación de los padres y la situación laboral de estos están correlacionadas con el aumento en la probabilidad de demandar educación posobligatoria en Colombia. Otros resultados muestran que existen diferencias estadísticamente significativas

* Ph. D. en Economía, profesor del Departamento de Economía, Universidad Icesi, Cali, Colombia. Tel: (57-2) 555 2334, Ext. 8844, Fax: (57-2) 555 1441. Correo electrónico: cggonzalez@ icesi.edu.co. Página web: www.icesi.edu.co/cggonzalez

+ PhD. en Economia. Profesor Titular del Departamento de Economía de la Universidad Icesi. Director del Observatorio Laboral del Observatorio Económico y Social del Valle del Cauca y Miembro del grupo de expertos en el Mercado Laboral del Ministerio de Trabajo de Colombia. Correo electrónico: jjmora@icesi.edu.co

$\ddagger 260$ WCOB, Department of economics, University of Arkansas, Fayetteville, AR 72701, EEUU. Correo electrónico: afcuadro@uark.edu

Para citar este artículo: González, C. G., Mora, J. J., \& Cuadros, A. F. (2014). Oportunidades educativas y características familiares en Colombia: un análisis por cohortes. Revista de Economía del Rosario, 17(1), 157-187. doi: dx.doi.org/10.12804/rev.econ.rosario.17.01.2014.06 
por ingresos, por cohortes y por género que agravan la desigualdad en las oportunidades educativas.

Clasificación JEL: I20, I23, I24.

Palabras clave: Educación, igualdad de oportunidades, Probit ordenado generalizado.

\title{
Family Characteristics and Educational Outcomes in Colombia: An Analysis by Cohorts
}

\begin{abstract}
This paper aims to determine which family characteristics are correlated with access to postcompulsory education in Colombia. To do this, generalized ordered probit model is estimated using seven cohorts with data from the Household Integrated Survey (GEIH) for 2008-2012. The main econometric results show that characteristics such as parental education, employment status and socioeconomic status are correlated with the increase in the probability of choosing post-compulsory education in Colombia. Therefore, there are significant differences that show the existence of inequality in educational opportunities.
\end{abstract}

JEL classification: I20, I23, I24.

Keywords: Education, inequality, generalized ordered probit.

\section{Oportunidades educativas e características familiares na Colômbia: uma análise por coortes}

\section{Resumo}

Este documento analisa a relação entre as características familiares e o acesso à educação pósobrigatória (meia e superior) na Colômbia. Para isso, estima-se um modelo probit ordenado generalizado usando sete coortes com os dados da Grande Enquete Integrada de Lares (GEIH) para o período 2008-2012. Os principais resultados econométricos mostram que características como a educação dos pais e a situação laboral deles estão correlacionadas com o aumento na probabilidade de demandar educação pós-obrigatória na Colômbia. Outros resultados mostram que existem diferenças estadisticamente significativas por estratos, por coortes e por gênero que agravam a desigualdade nas oportunidades educativas.

Classificação JEL: I20, I23, I24.

Palavras-chave: Educação, igualdade de oportunidades, probit ordenado generalizado. 


\section{Introducción}

En la primera década del siglo XXI, Colombia (o la sociedad colombiana) ha experimentado una expansión del acceso al sistema educativo, tanto para los niveles obligatorios como para los niveles posobligatorio (Acevedo, Zuluaga y Jaramillo, 2008; González, 2011; Ramírez, 2008). Esta expansión puede ser analizada empleando la tasa de cobertura bruta, principal indicador utilizado para medir la cobertura educativa, definida como la relación porcentual entre los alumnos matriculados en un nivel de enseñanza específico (independiente de la edad que estos tengan) y el total de la población en el rango de edad teórico correspondiente a dicho nivel. Según datos del Ministerio de Educación Nacional (MEN), institución encargada de regular la educación en Colombia, la educación obligatoria, que comprende un año de preescolar y nueve años de educación básica, presenta tasas de cobertura superiores al 100\% para el año 2011.

Adicionalmente, la tasa de cobertura bruta para básica primaria es de $117 \%$ y para básica secundaria es de $104 \%$. Sin embargo, el resultado para la educación posobligatoria, que comprende la educación media y la educación superior, ha sido poco satisfactorio en comparación con los niveles anteriores. Por ejemplo, la tasa de cobertura bruta para la educación media es del 79\% y para la educación superior (técnica, tecnológica y universitaria) es tan solo del $37 \%$ en 2011 (MEN, 2012).

Sumado a lo anterior, si se comparan los resultados de la tasa de cobertura bruta de la educación superior con el contexto internacional, Colombia resulta ser un país rezagado en el tema. Para analizar el contexto internacional, se utiliza el coeficiente de Gini (un indicador de desigualdad de ingresos), pues es un buen índice de comparación del bienestar económico del individuo medio entre países. Utilizando este indicador, junto a la tasa de cobertura bruta para la educación superior, se puede observar en la figura 1 que el grado de desigualdad pareciera estar afectando los objetivos de cobertura en la educación posobligatoria para Colombia, dado que países que presentan un menor coeficiente, es decir, una mayor medida de igualdad, presentan tasas de cobertura mayores. $\mathrm{Al}$ respecto, cabe señalar que Colombia presenta el coeficiente de Gini más alto, el mayor grado de desigualdad y una de las menores tasas de cobertura en educación superior para el año 2010. 


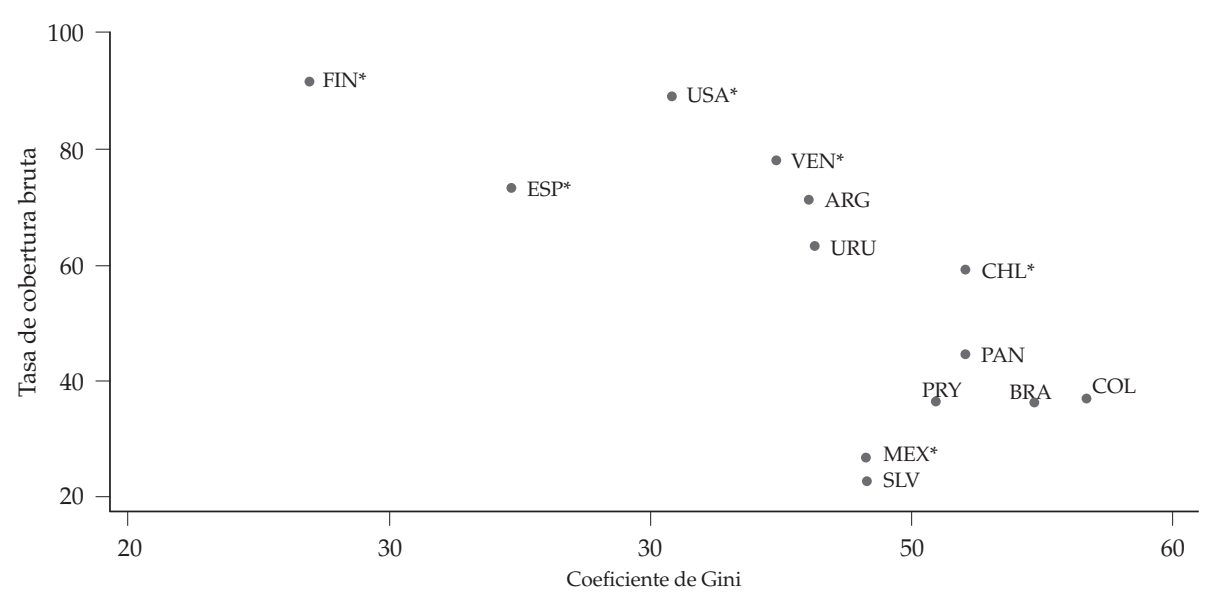

Figura 1. Tasa de cobertura bruta de la educación superior vs. coeficiente de Gini 2010 Fuente: Elaboración propia con datos del PNUD y MEN.

Nota: TCB (Tasa de Cobertura Bruta) tomada del MEN y coeficiente de Gini tomado del (PNUD). *Gini para el último año disponible.

En el marco internacional, algunos autores como Evans y Schwab (1995), Sanders y Krautmann (1995), Card y Krueger (1996) y Neal (1997) sugieren que las oportunidades educativas en los niveles posobligatorios están fuertemente relacionadas con el entorno familiar, el entorno socioeconómico y las características propias del individuo. Por lo tanto, si estas características de la población persisten en el transcurso del tiempo, se corre el riesgo de que estas disparidades se transfieran a futuras generaciones (Di Paolo, 2012).

Habría que mencionar también que para entender algunos de los principales aspectos relacionados con las desigualdades existentes en el sistema educativo colombiano es necesario contextualizar el marco institucional. El sistema educativo colombiano está amparado por el artículo 67 de la Constitución Política de 1991, el cual tiene como objetivo lograr un sistema educativo en el que participe toda la sociedad. Asimismo, la Ley 30 de 1992 (ley de educación superior) y la Ley 115 de 1994 (ley de educación general) garantizan la equidad en el acceso y la oportunidad de alcanzar el mayor nivel de educación, sin importar el origen social y el entorno familiar de los individuos.

De acuerdo con este contexto legal, en el país existen unos niveles de educación que son obligatorios y otros que son posobligatorios. Igualmente, los niveles bajos son gratuitos y los más altos no. Por ejemplo, la educación superior no es obligatoria y tampoco es gratuita, es el individuo o su familia quienes deciden si demandar o no estos niveles de educación. Esta particularidad del sistema 
de educación colombiano radica en que el sistema educativo colombiano está estratificado con una separación a los quince años de edad, dado que por mandato constitucional la educación es obligatoria entre los cinco y los quince años. En otras palabras, una vez los individuos alcanzan esta edad o los nueve años de educación obligatoria, ellos, o sus padres, deben decidir si acceden (o no) a la educación media y, posteriormente, a la educación superior, ambas son elecciones de educación posobligatorias. Hanushek y Wöbmann (2006), Brunello y Checchi (2007), entre otros, resaltan que esta clase de separación entre niveles obligatorios y posobligatorios refuerza la correlación que existe entre las características familiares y el acceso a la educación.

Por todo lo anterior, este artículo tiene como objetivo analizar la relación entre las características familiares y la elección por educación posobligatoria por cohortes de edad de los jóvenes en Colombia. Lo anterior, permitirá analizar si existen diferencias estadísticamente significativas que muestren la existencia de una desigualdad de oportunidades educativas, principalmente, por cohortes de nacimiento.

Este artículo está compuesto por siete secciones, incluyendo esta primera parte. En la segunda sección, se hace una revisión de la literatura relacionada con las elecciones educativas donde se discuten los efectos de las características familiares y personales observadas. En la tercera sección se muestra un análisis descriptivo de los datos y de las variables utilizadas. En la cuarta sección se discute la metodología econométrica utilizada. En la quinta sección se exponen los principales resultados encontrados. Finalmente, se presenta una sección de conclusiones.

\section{Revisión de literatura}

La literatura en economía de la educación ha experimentado una gran expansión a partir de los trabajos de Becker (1964) y Mincer (1974), siendo la teoría del capital humano su centro de atención (Hanushek y Woessmann, 2008). Estos trabajos identificaron que existe un vínculo entre las ganancias de un individuo en su ciclo de vida y la decisión de invertir en capital humano, por lo cual esta última dependerá de los retornos esperados y de los costos de invertir. En esta misma línea, Willis y Rosen (1979) muestran la influencia positiva de las ganancias esperadas en el ciclo de vida de los jóvenes y la decisión de ir a la universidad. Además, muestran cómo los ingresos no percibidos, debido a la participación en la educación, representan un costo de oportunidad adicional para los individuos, por lo cual se espera que mayores niveles de ingresos 
percibidos se asocien con una menor participación en el sistema educativo ( $\mathrm{Fu}-$ ller, Manski y Wise, 1982; Van Tilberg y Dubois, 1989).

Por otra parte, Leslie y Brinkman (1987) y Héller (1997) estudian los gastos de matrícula, los cuales proveen otros costos que afectan la participación del individuo en educación, y encuentran que gastos mayores en matrícula afectarán negativamente el acceso. Con respecto a los factores personales y familiares, Osterbeek y Van Ophem (2000) construyeron un modelo estructural de elecciones de educación que incluye la distancia relativa para educarse. Ellos encuentran que las preferencias para educarse varían con respecto al entorno social. Así, un nivel de educación mayor en la madre está asociado con un nivel mayor de preferencia por educarse de los hijos. Albert (1998) encuentra que las características familiares, sobre todo la educación de los padres, son elementos importantes en la educación superior en España. Rahona (2006) encuentra que, a pesar del proceso de apertura de la universidad española, características como la educación de los padres, continúan influyendo en la realización de estudios universitarios.

Desde los factores sociales, Clancy $(1997,2001)$ muestra que aquellos que acceden a educación superior en Irlanda provienen, principalmente, de clases sociales superiores. En esta misma línea, Whelan y Hannan (1999) al examinar el rol de las clases sociales en la transición a varios niveles de educación, encuentran que la transición de secundaria alta al nivel de educación terciario es significativamente afectada por la clase social de los padres. Además, estos estudios resaltan el rol de los beneficios y costos asociados con el nivel de educación terciaria y la decisión de los jóvenes de acceder o no a este nivel. En este punto, los beneficios pueden ser vistos como las ganancias potenciales, mientras los costos son directos e indirectos. Los costos directos incluyen gastos de matrícula y el principal costo indirecto es el ingreso que el individuo deja de recibir por estar fuera del mercado laboral.

En lo que respecta a la influencia de factores económicos, Hayeman y Wolfe (1995) concluyen que menores niveles de ingresos de los padres están correlacionados con menores niveles de educación de los hijos. Por lo cual, se puede observar que hogares con bajos ingresos tendrán un impacto negativo en la decisión de mayores niveles de educación. Ackemoglu y Pischke (2001) soportan esto usando el cambio en la distribución del ingreso familiar, para estimar el impacto del ingreso del padre en la decisión de educarse de sus hijos. Ellos encuentran que un incremento del ingreso familiar está asociado con una mayor probabilidad de participar en niveles de educación más altos. Por su parte, Cameron y Heckman (1999) discuten el impacto de la restricción de crédito que enfrentan las familias con ingresos bajos. 
La condición del mercado laboral también cumple un papel importante en la influencia de acceder a niveles educativos más altos, al afectar la decisión de permanecer en el sistema educativo cuando el mercado laboral se deprime. Gustman y Steinmeier (1981), Light (1995), Rice (1999) y Giannelli y Monfardini (2003) discuten este tema desde el costo de oportunidad, asociado con la educación superior. En este sentido, si hay muchas oportunidades de trabajo para los jóvenes, estos preferirán rechazar los niveles de educación superior a favor de participar en el mercado laboral.

Otros trabajos relevantes discuten la transición de la educación al mercado laboral. Por una parte, Mondrego (1987), Albert, Toharia y Davia (2009) encuentran que una mayor educación de las mujeres frente a los hombres no afecta su posición en la inserción en el mercado laboral. Asimismo, encuentran que la titulación alcanzada por los jóvenes influye en la decisión de salir del sistema educativo. Por otra parte, Albert y Davia (2010) encuentran que el logro educativo en la educación obligatoria es determinante en las trayectorias que los jóvenes siguen entre el sistema educativo y el mercado laboral.

Para Colombia se resaltan los trabajos de Sánchez y Núñez (2003), quienes examinan la estructura de los hogares colombianos, los retornos a la educación y el capital humano, al utilizar un enfoque por cohortes y encuentran que el capital humano ha sido acumulado de manera desigual por los distintos grupos de ingresos. Además, la nueva generación de mujeres ha alcanzado mayores niveles de educación que los hombres. En la misma línea, González, Girón y Sánchez (2003), encuentran estos resultados para Cali.

Por su parte, Castillo (2007) y Mora (2008) discuten el fenómeno de sobreeducación en Colombia. El primero, resalta una relación estrecha entre los puestos de trabajo y la cualificación de las personas. El segundo, concluye que la probabilidad de ser más educado depende de la movilidad entre las diferentes áreas de desempeño, experiencia, sexo, tamaño de la empresa y el grado de educación. Igualmente, Mora (2008) expone que algunos trabajadores ganan un $2 \%$ menos que los trabajadores correctamente educados.

Por otro lado, Tenjo (2002) resalta un crecimiento moderado de la matrícula universitaria y la necesidad de reformas educativas y en la distribución del ingreso. Ramírez (2008), al utilizar modelos de elección discreta, realiza una caracterización cuantitativa de la demanda de cupos universitarios por parte de bachilleres egresados de ciudades intermedias y encuentra que el número de programas ofrecidos aumenta la probabilidad de selección de las universidades por parte de los bachilleres; mientras que el ranking universitario está inversamente relacionado con dicha probabilidad. También, encuentra que la posición socioeconómica, medida por medio del ingreso del hogar, es una variable bastante explicativa de la elección universitaria. 
Sumado a lo anterior, Acevedo et al. (2008) sugieren que la oferta educativa tiene una gran influencia sobre las tasas de matrícula en las instituciones de educación superior. Igualmente, encuentran que la dinámica del empleo desempeña un papel significativo en las decisiones de ingresar en estudios superiores. Con respecto a esta relación entre demanda de educación y mercado laboral, Mora y Muro (2008) muestran que los individuos con títulos en educación superior presentan ganancias adicionales en sus salarios.

Adicionalmente, González (2011) y Sánchez y Zúñiga (2012) resaltan que aunque la cobertura en educación superior creció, lo hizo también la tasa de deserción. Así, encuentran que el incremento en el abandono está explicado por la mayor vulnerabilidad tanto socioeconómica como académica de las nuevas cohortes de estudiantes.

Otro aspecto que se discute en la literatura colombiana es la calidad. Barrera, Maldonado y Rodríguez (2012) resaltan que, si bien existe un aumento en la cobertura, se debe desarrollar una política educativa para Colombia que permita mejorar la calidad de la educación básica, secundaria y media, y así convertirla en una herramienta efectiva de movilidad social. Finalmente, Ramírez (2008), utilizando modelos de elección discreta, encuentra que la posición económica, medida como el ingreso del hogar, es significativa a la hora de explicar las elecciones educativas. De esta forma, los bachilleres provenientes de hogares con ingresos bajos tienden a optar por mejores niveles de educación.

\section{Datos y estadísticas descriptivas}

En este documento, se utilizan los datos del 2008 al 2012 de la Gran Encuesta Integrada de Hogares (GEIH), realizada por el Departamento Administrativo Nacional de Estadística (DANE). El uso de estos cinco años de la Encuesta es apropiado para analizar la relación entre las oportunidades educativas y las características familiares, porque contiene información sobre las características del individuo, la educación de los padres y otras características familiares.

La información de la GEIH permite agrupar el acceso educativo en seis categorías: sin educación/analfabeta, preescolar, primaria, secundaria, educación media y educación superior. Esta información aplica tanto para la educación del individuo como para el mayor nivel de educación completado por los padres, lo cual representa el entorno educativo de los integrantes del hogar. Además, esta encuesta es homogénea para el periodo seleccionado y permite crear siete cohortes de nacimiento que se observan en la tabla 1, cada una de cuatro años. La importancia de construir las cohortes en un corte transversal radica en que 
permiten analizar la dinámica del proceso educativo en el tiempo. También, se utiliza en este documento información de la oferta de educación superior proveniente del ministerio de educación nacional. Algunas variables que se recogieron son el número de alumnos matriculados y el número de instituciones de educación superior. La variable que se incluyó en el análisis fue el cociente entre estas dos para así medir la oferta disponible de cupos.

Tabla 1. Cohortes de nacimiento

\begin{tabular}{lccccc}
\hline \multicolumn{5}{c}{ Rango de años } \\
\hline C1 & 2008 & 2009 & 2010 & 2011 & 2012 \\
\hline C2 & $1991-1994$ & $1990-1993$ & $1989-1992$ & $1988-1991$ & $1987-1990$ \\
C3 & $1990-1993$ & $1989-1992$ & $1988-1991$ & $1987-1990$ & $1986-1989$ \\
C4 & $1989-1992$ & $1988-1991$ & $1987-1990$ & $1986-1989$ & $1985-1988$ \\
C5 & $1988-1991$ & $1987-1990$ & $1986-1989$ & $1985-1988$ & $1984-1987$ \\
C6 & $1987-1990$ & $1986-1989$ & $1985-1988$ & $1984-1987$ & $1983-1986$ \\
C7 & $1986-1989$ & $1985-1988$ & $1984-1987$ & $1983-1986$ & $1982-1985$ \\
\hline & $1985-1988$ & $1984-1987$ & $1983-1986$ & $1982-1985$ & $1981-1984$ \\
\hline C1 & $16-19$ & $17-20$ & $18-21$ & $19-22$ & $20-23$ \\
C2 & $17-20$ & $18-21$ & $19-22$ & $20-23$ & $21-24$ \\
C3 & $18-21$ & $19-22$ & $20-23$ & $21-24$ & $22-25$ \\
C4 & $19-22$ & $20-23$ & $21-24$ & $22-25$ & $23-26$ \\
C5 & $20-23$ & $21-24$ & $22-25$ & $23-26$ & $24-27$ \\
C6 & $21-24$ & $22-25$ & $23-26$ & $24-27$ & $25-28$ \\
C7 & $22-25$ & $23-26$ & $24-27$ & $25-28$ & $26-29$ \\
\hline
\end{tabular}

Fuente: Elaboración propia.

Por otra parte, la estructura del sistema educativo colombiano se presenta en la figura 2, que se divide en educación obligatoria y posobligatoria. En la figura se puede observar que un individuo debe tomar obligatoriamente un año de preescolar y nueve años de educación básica. La educación básica se encuentra dividida en dos niveles: el primer nivel es la educación básica primaria, que comprende cinco años de educación, seguido por la educación básica secundaria, conformada por cuatro años.

Una vez el individuo alcanza los nueve años de educación, él o su familia deciden si acceden a la educación media, que es el primer nivel posobligatorio. 
Este nivel está conformado por el servicio social de educación especial y la educación media académica y media técnica. Estos dos últimos niveles de educación media comprenden dos años de educación y otorgan el título de bachiller, que es el que permite continuar en el proceso educativo hacia la educación superior.

No obstante, fuera de obtener el título de bachillerato, para ingresar a la educación superior es necesario presentar la prueba de Estado Saber 11, que tiene como fin comprobar el grado de desarrollo de competencias de los estudiantes de undécimo grado. Una vez el individuo cumple estos requisitos, él o su familia deben decidir si accede (o no) a la educación superior. La educación superior es la segunda elección posobligatoria y se divide en técnica, tecnológica y universitaria. La educación técnica comprende dos años de educación y forma al individuo en ocupaciones de carácter operativo e instrumental. La educación tecnológica comprende tres años de educación y ofrece programas de formación en ocupaciones y de formación académica en profesiones o disciplinas. La educación universitaria comprende cinco años de educación. Según datos del MEN, existe una preferencia de la educación universitaria sobre la educación técnica y tecnológica. Por ejemplo, la educación universitaria tiene una participación de la matrícula total del $62,6 \%$, mientras que la educación tecnológica y técnica tienen una participación del $27,4 \%$ y $4,1 \%$, respectivamente.

Después de presentar el marco institucional y algunas de las principales características del sistema de educación en Colombia, es necesario analizar las estadísticas descriptivas de las variables que condicionan la demanda de educación, empezando por la educación de los padres.

Algunos trabajos analizan la demanda de educación de los hijos en función de la demanda de educación de los padres y encuentran que el nivel de educación de los padres influye en general en la demanda de educación de los hijos (Albert, 1998; Leibowitz, 1974; Rahonna, 2006; Valiente, 2003;). Estos trabajos resaltan la importancia de analizar la educación de los padres por su fuerte relación con el ingreso familiar, el ambiente intelectual en el que vive el estudiante y la habilidad de los hijos. De igual manera, Lillard y Robert (1994) encontraron que la educación del padre influye con mayor fuerza en la educación de los hijos, mientras Lauer (2003) y Chevalier y Lanot (2002), resaltan la educación de la madre, al relacionar el éxito académico con el cuidado y la atención que esta les brinda. En este sentido, es importante conocer la distribución de la educación de los padres en el sistema educativo colombiano. 


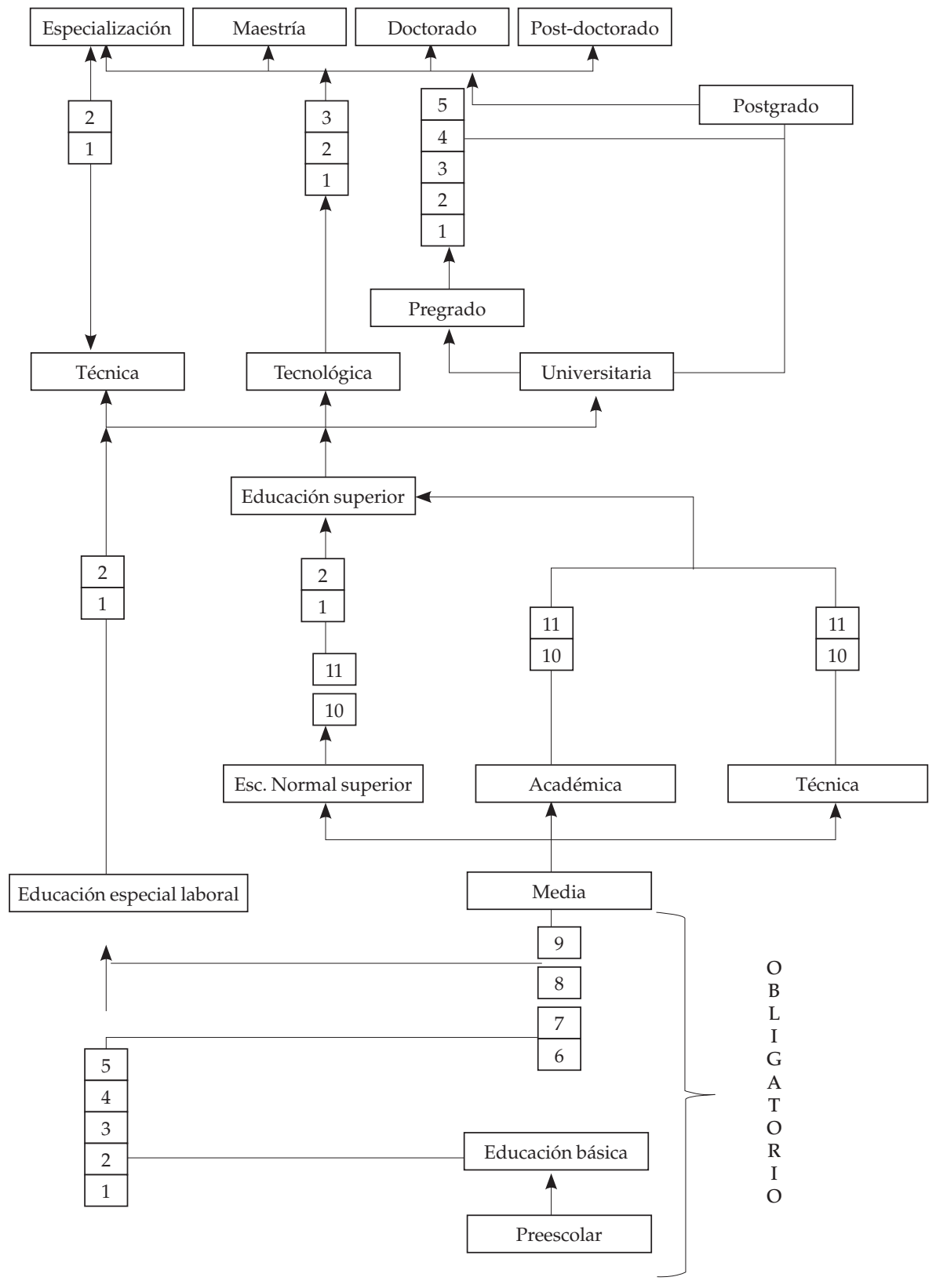

Figura 2. Sistema de educación colombiano

Fuente: UNESCO. 
Los datos de la GEIH permiten observar en la figura 3 una imagen descriptiva de la brecha educativa, asociada a la educación familiar, y su evolución entre cohortes. Esta figura muestra una importante brecha que persiste en el tiempo, donde los individuos de las cohortes más jóvenes tienen padres cada vez con mayores niveles educativos. Se puede resaltar la reducción de los padres sin educación/analfabetas para las cohortes más viejas en el 2009 y 2010 y su desaparición en las cohortes más jóvenes para el 2011 y el 2012. Con respecto a la educación primaria (obligatoria) y la educación media y superior (posobligatorias), se resalta para las cohortes 1, 2 y 3 una reducción de los padres con educación primaria y un mayor porcentaje con educación superior. Por su parte, para las cohortes 4, 5 y 6, aunque el porcentaje de padres con educación primaria no ha presentado una variación importante, los padres con educación media sí han disminuido y han dado paso a un mayor porcentaje de padres con educación superior.

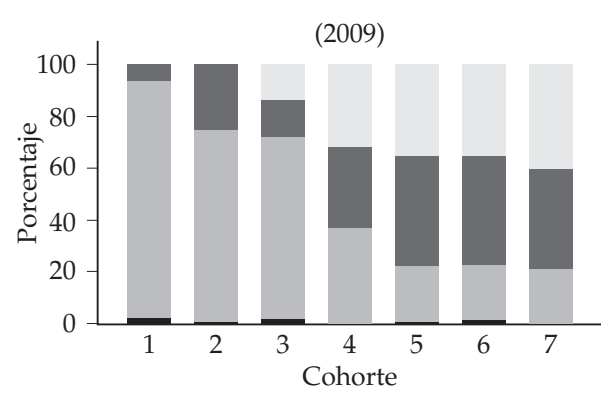

Sin estudios Primaria $\square$ Media $\square$ Superior

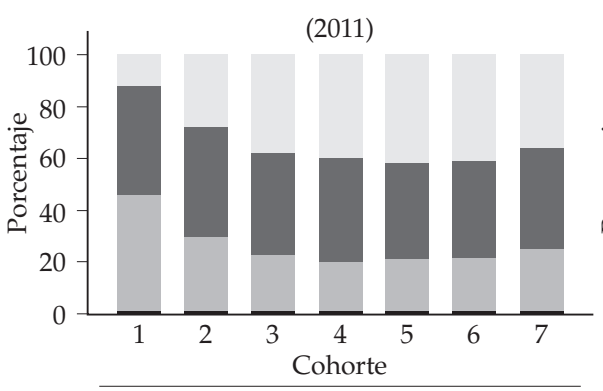

Sin estudios Primaria $\square$ Media Superior

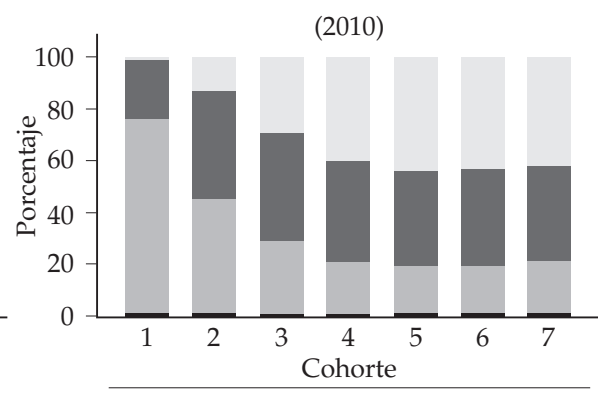

Sin estudios Primaria Media Superior

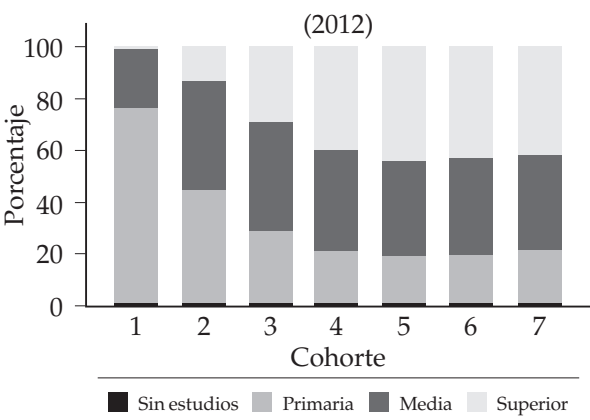

Figura 3. Distribución niveles de educación de los padres por cohorte Fuente: Elaboración propia con GEIH.

Nota: 1, 2, 3, 4, 5, 6 y 7 corresponden a las cohortes de nacimiento. 
Estos resultados se confirman en la tabla 2, donde se analizan los estudios del padre y la madre por separado. En la tabla se puede observar que existe una mayor participación de los padres con estudios de primaria y un comportamiento creciente en la proporción con educación media durante los cuatro años de análisis. Por otra parte, la categoría variable ser madre concentra sus estudios en la educación primaria, seguida por media y superior, todas con un comportamiento creciente. De otra parte, se resalta el bajo porcentaje de padre y madre analfabeta.

Es importante considerar la presencia de otras variables que pueden ser determinantes del acceso a mayores niveles educativos, dado que la brecha en los niveles de educación puede ser producto de otros determinantes importantes como factores personales, factores de origen social o factores socioeconómicos, entre otros. Con la información disponible en la GEIH se definen un conjunto de características familiares, socioeconómicas y del individuo que se encuentran en la tabla 2 con sus respectivas estadísticas descriptivas.

Una de las características personales que se usará en este trabajo es la variable género. Recientemente, diversos trabajos (Kodde \& Ritzen, 1988; Rahona, 2006; Rice, 1987; San Segundo, 2003) han mostrado que las mujeres presentan una mayor participación en las aulas que los hombres; por lo cual es relevante conocer la importancia del género en el acceso a la educación en Colombia. En este sentido, existe una mayor proporción de las mujeres durante los cuatro años, la cual se mantiene alrededor del $52 \%$.

Otros factores de origen social, como la actividad del padre y de la madre, pueden ser vistos como el reflejo del estatus socioeconómico de la familia (Albert, 1998) y como un determinante de la inversión en educación (Albert, 2000). La actividad del padre se relaciona con la capacidad financiera a la que se enfrenta la familia, mientras la importancia de la actividad de la madre está relacionada con la permanencia en el hogar y el cuidado de los hijos ( $\mathrm{O}^{\prime}$ Brien \& Jones, 1999). Para Colombia, se observa un crecimiento en la participación de los padres en el mercado laboral, se puede apreciar que la actividad del padre se concentra en ocupados, con un comportamiento creciente, mientras se presenta un comportamiento decreciente de los padres inactivos. Por su parte, el porcentaje de madres se concentra en inactivos, con un comportamiento creciente, tanto en este rubro como en las madres ocupadas y una disminución en las desocupadas.

Para las características de la familia, como el tamaño del hogar y el número de hermanos menores de 16 años, variables que muestran la composición de las familias colombianas y que reflejan los límites financieros y la carga económica (Hanushek, 1992; Petrongolo y San segundo, 1998; Rahonna, 2006; Valiente, 
2003), se aprecia que cada vez es mayor el porcentaje de hogares con menor número de personas y con menor número de hermanos menores de 16 años. Otra variable relevante es la región, ya que en Colombia, la capital Bogotá es un distrito especial que concentra gran parte de los recursos de la educación superior.

También se observan otros factores que pueden influir en la demanda de mayores niveles educativos, como la tasa de desempleo juvenil que se ha mantenido en alrededor del $20 \%$, entre 2008 y 2011, bajando en 2012 al 16\%, y la tasa de inactividad juvenil que pasó del 38,6\% al 36,55\% de 2008 a 2012. Otra característica es el ingreso del hogar que se ha organizado en tres categorías (i. menos de un Salario Mínimo Legal Vigente (SMLV), ii. entre 1 y 2 SMLV y iii. más de $2 \mathrm{SMLV}$ ), esta variable muestra que aproximadamente el $45 \%$ de la población tiene menos de un SMLV.

Por último, se tienen en cuenta las variables de la oferta educativa. En este caso, se construyó un indicador de oferta por medio del cociente entre el número de alumnos matriculados en la educación superior, dividido en el número de Instituciones de Educación Superior (IES), este indicador muestra cómo la oferta ha ido creciendo entre los años 2008 y 2012. Esta información proviene del Ministerio de Educación Nacional, cuya última actualización corresponde al año 2012.

Tabla 2. Estadísticas descriptivas

\begin{tabular}{llrrrrr}
\hline & Variable & \multicolumn{5}{c}{ Porcentajes } \\
\cline { 3 - 7 } & & 2008 & 2009 & 2010 & 2011 & \multicolumn{1}{c}{2012} \\
\hline Género & Mujeres & 52,86 & 53,26 & 52,47 & 52,19 & 52,73 \\
\hline \multirow{2}{*}{$\begin{array}{l}\text { Situación res- } \\
\text { pecto padres }\end{array}$} & Sin padre & 30,21 & 32,51 & 32,41 & 35,85 & 36,14 \\
& No es hijo & 15,19 & 15,78 & 16,14 & 18,28 & 15,01 \\
& Hijo único & 5,02 & 6,98 & 7,02 & 9,25 & 10,24 \\
& Analfabeta/sin estudios & 2,03 & 2,27 & 2,07 & 1,72 & 2,06 \\
Educación del & Primaria & 17,25 & 19,73 & 18,06 & 17,09 & 15,53 \\
padre & Secundaria & 10,15 & 11,76 & 11,76 & 9,75 & 9,87 \\
& Media & 14,72 & 17,81 & 17,25 & 16,17 & 15,32 \\
& Educación superior & 10,69 & 10,14 & 10,83 & 10,66 & 10,87 \\
\hline \multirow{2}{*}{$\begin{array}{l}\text { Educación de la } \\
\text { madre }\end{array}$} & Analfabeta/sin estudios & 2,99 & 3,57 & 2,97 & 2,90 & 3,05 \\
& Primaria & 25,96 & 28,38 & 27,42 & 24,01 & 22,68 \\
& Secundaria & 16,62 & 17,18 & 17,43 & 16,15 & 15,11 \\
\hline
\end{tabular}




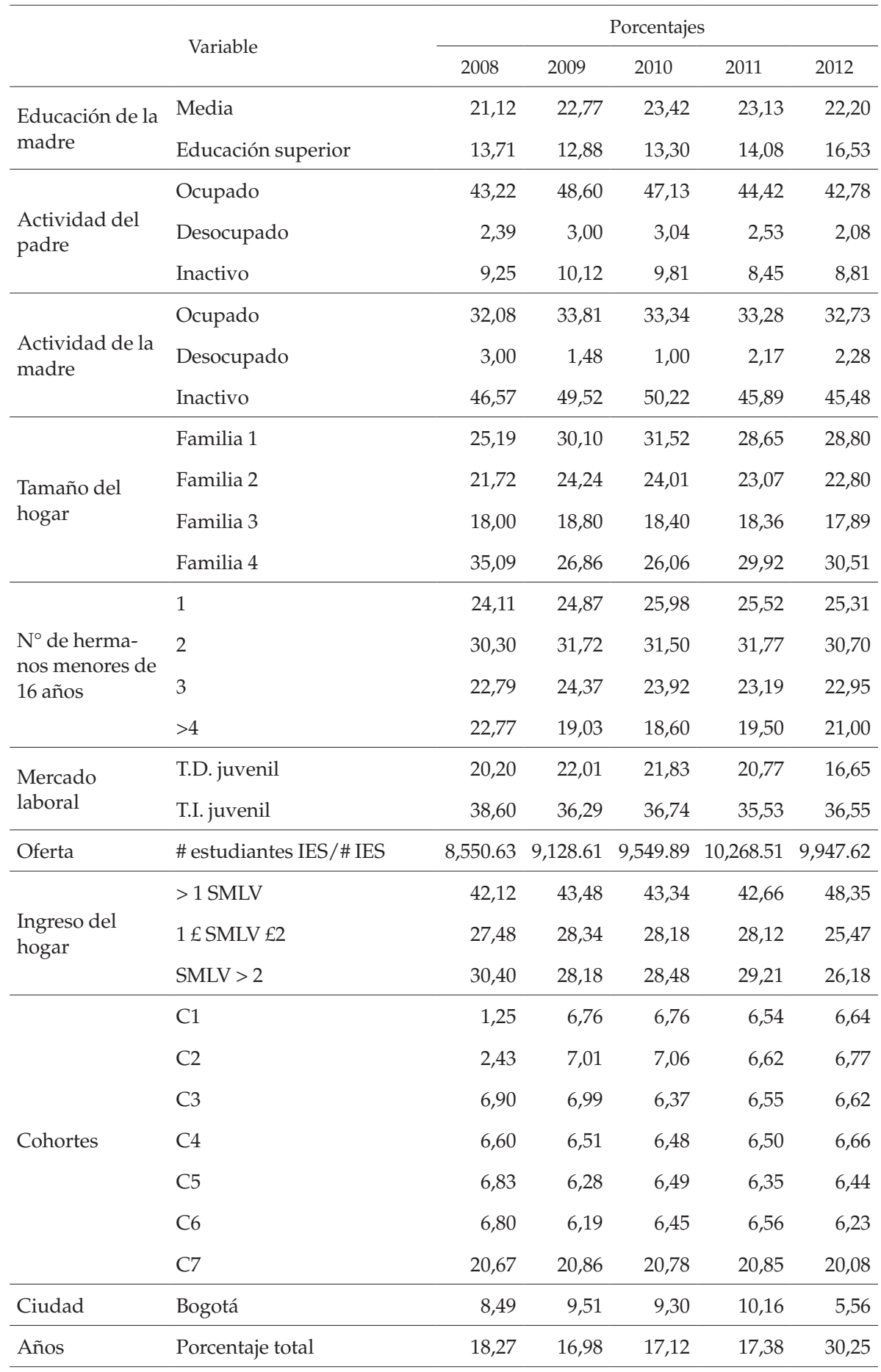

Fuente: Elaboración propia con GEIH y datos del Ministerio de Educación. 


\section{Metodología econométrica}

De acuerdo con el sistema de educación colombiano, la información disponible de la GEIH y la teoría del capital humano, las elecciones educativas en Colombia pueden ordenarse en cuatro categorías: i. Analfabeta/sin estudios, ii. Educación básica, iii. Educación media y iv. Educación superior. La especificación más recomendable para este tipo de variable dependiente discreta es un probit ordenado (Cameron y Heckman, 1998; Chevalier y Lanot, 2002; Ermisch y Francesconi, 2001; Lauer, 2003). Por este motivo, inicialmente, se estimaron los modelos como probit ordenados. Sin embargo, después de realizar el contraste de regresiones paralelas y dado el valor de la Chi-cuadrado se decidió estimar los modelos como un probit ordenado generalizado, lo cual garantiza mejores propiedades estadísticas en los estimadores (Greene, 2012).

Con el objetivo de obtener una representación temporal, se estiman dos modelos, el primero con cada cohorte como variable independiente y un segundo modelo para cada cohorte, manteniendo las características familiares y del individuo fijas. Es importante resaltar que los resultados de la estimación de modelos de elección discreta no son fácilmente interpretables por lo que se aconseja utilizar el cálculo de los efectos marginales y de las probabilidades condicionales. Por lo tanto, a continuación, se muestran en todas las tablas los efectos marginales y en las figuras las probabilidades asociadas a cada elección educativa.

\section{Principales resultados}

De acuerdo con la teoría del capital humano, las características familiares se consideran uno de los principales determinantes de la demanda de educación (Freeman, 1986; Ordovensky, 1995). De este modo, la probabilidad de que una persona demande mayores niveles de educación estará en función de diferentes variables personales, familiares y socioeconómicas.

En la tabla 3, la significancia de los coeficientes indica que existen diferencias estadísticas en las características personales, familiares y del entorno socioeconómico de los individuos. Por ejemplo, los resultados muestran que el ser mujer incrementa la probabilidad de acceder a los niveles de educación superior. Este resultado concuerda con la literatura citada en este artículo, donde se resalta el aumento extraordinario que han experimentado las mujeres en la participación en el sistema educativo. Al mismo tiempo, González (2011) sugiere que esto puede estar relacionado con las reformas educativas como la Ley 30 de 1992 o con la situación de la mujer en el mercado laboral, debido a 
que estas presentan mayores tasas de desempleo y una fuerte discriminación, lo que puede estar fortaleciendo su acceso al sistema educativo para compensar en un futuro la discriminación laboral por el hecho de ser mujeres (Caicedo \& Mora, 2013).

Por otra parte, se resaltan los resultados para la educación de los padres, el tamaño del hogar y el número de hermanos menores de dieciséis años, características que generan diferencias significativas. Con respecto a las características de los padres, entre mayor sea la educación del padre y la madre, mayor será la probabilidad de demandar mayores niveles educativos. Así, tener padres con educación media reduce la probabilidad de quedarse sin estudios y alcanzar solo la educación primaria, pero aumenta la probabilidad de alcanzar estudios posobligatorios. En la misma forma, tener madre con educación primaria aumenta la probabilidad de alcanzar la educación obligatoria, pero reduce la probabilidad de alcanzar estudios en educación media y superior. No obstante, tener secundaria o alcanzar niveles posobligatorios aumenta la probabilidad de que los hijos accedan a estos mismos niveles. Por otra parte, en lo que respecta a la participación en el mercado laboral, tener padre y madre desempleados disminuye la probabilidad de que los individuos demanden educación media y superior, ambas posobligatorias.

Para la estructura del hogar, se resalta que si bien el tamaño del hogar no disminuye la probabilidad de demandar niveles de educación media y superior, el número de hermanos menores de dieciséis años sí lo hace. Esto puede estar explicado en el hecho de que ambos niveles posobligatorios implican una carga financiera para el hogar. Es decir, no es el número de miembros lo que influye en el acceso; sino de estos cuáles pueden acceder al sistema educativo.

Con respecto a otros factores que pueden afectar la demanda de educación, el ingreso del hogar es una variable que muestra que un ingreso bajo reduce la probabilidad de demandar educación en los niveles más altos; mientras que los jóvenes que provienen de hogares con ingresos altos tiene una probabilidad positiva y creciente en el tiempo. Por su parte, la variable Bogotá (que recoge a los individuos que se encuentran en el distrito capital) refleja la existencia de diferencias significativas y, además, indica que los individuos que viven en la capital del país tienen una mayor probabilidad de demandar educación posobligatoria.

Asimismo, se incluyeron las cohortes y el tiempo y se encuentra que existen diferencias estadísticamente significativas entre ellos. Para las cohortes, en cada una de ellas, el efecto marginal de quedarse sin estudios o acceder a la educación primaria es negativo. Sin embargo, cada cohorte tiene un efecto marginal positivo en el acceso a la educación posobligatoria. Para los años 2008, 2009 y 2010, el efecto marginal de quedarse sin estudios o demandar educación obligatoria 
es negativo, mientras demandar los niveles posobligatorios, media y superior, tiene un efecto positivo.

Adicionalmente, la variable de oferta de educación superior incluida tiene un efecto positivo y significativo sobre la probabilidad de demandar educación superior. Por esta razón, la oferta de cupos afecta positivamente las elecciones educativas por los niveles de educación superior. Por otro lado, de las variables relativas a las condiciones del mercado de trabajo solo resulta significativa la tasa de inactividad juvenil que tiene un efecto negativo y significativo sobre la probabilidad de demandar educación superior. La tasa de desempleo juvenil no resulta significativa y, aunque su signo positivo coincide con la literatura, una tasa de desempleo juvenil creciente aumenta en los jóvenes las probabilidades de continuar en el sistema educativo.

Finalmente, estos resultados sugieren que para Colombia las elecciones de demandar mayores niveles educativos están condicionadas a la dotación de las características personales, familiares, de la estructura del hogar, condiciones de oferta y de la cohorte de nacimiento del individuo.

Tabla 3. Efectos marginales elecciones educativas por niveles

\begin{tabular}{|c|c|c|c|c|c|}
\hline & Variables & $\begin{array}{c}\text { Sin } \\
\text { estudios }\end{array}$ & Primaria & Media & Superior \\
\hline Género & Mujer & $-0,0022^{* * *}$ & $-0,0820^{* * *}$ & $-0,0087^{* * *}$ & $0,0931^{* * *}$ \\
\hline \multirow{4}{*}{$\begin{array}{l}\text { Situación respecto } \\
\text { de los padres }\end{array}$} & Sin padre & 0,8571 & $-0,0091^{*}$ & $-0,0054^{*}$ & $-0,0251^{* * *}$ \\
\hline & Sin madre & 0,0254 & 0,0001 & $0,0071^{*}$ & $0,0145^{* *}$ \\
\hline & No es hijo & 0,0965 & $-0,0055$ & $-0,015^{*}$ & $-0,0241^{* *}$ \\
\hline & Hijo único & 0,0021 & $0,0004^{*}$ & $0,0002^{*}$ & $0,0541^{* * *}$ \\
\hline \multirow{4}{*}{$\begin{array}{l}\text { Educación del } \\
\text { padre }\end{array}$} & Primaria & $-0,0003^{* * *}$ & $-0,0140^{* * *}$ & $-0,0019^{* * *}$ & $0,0162^{* * *}$ \\
\hline & Secundaria & $-0,0005^{* * *}$ & $-0,0236^{* * *}$ & $-0,0036^{* * *}$ & $0,0279^{* * *}$ \\
\hline & Media & $-0,0020^{* * *}$ & $-0,0943^{* * *}$ & $-0.0251^{* * *}$ & $0,1215^{* * *}$ \\
\hline & Superior & $-0,0028^{* * *}$ & $-0,1597^{* * *}$ & $-0,0732^{* * *}$ & $0,2359^{* * *}$ \\
\hline \multirow{4}{*}{$\begin{array}{l}\text { Educación de la } \\
\text { madre }\end{array}$} & Primaria & $0,0002^{* * *}$ & $0,0103^{* * *}$ & $0,0010^{* * *}$ & $-0,0116^{* * *}$ \\
\hline & Secundaria & $-0,0004^{* * *}$ & $-0,0185^{* * *}$ & $-0,0026^{* * *}$ & $0,0217^{* * *}$ \\
\hline & Media & $-0,0026^{* * *}$ & $-0,1217^{* * *}$ & $-0,0333^{* * *}$ & $0,1577^{* * *}$ \\
\hline & Superior & $-0,0038^{* * *}$ & $-0,2167^{* * *}$ & $-0,1223^{* * *}$ & $0,3429^{* * *}$ \\
\hline \multirow{2}{*}{$\begin{array}{l}\text { Actividad del } \\
\text { padre }\end{array}$} & Inactivo & $0,0026^{* * *}$ & $0,0922^{* * *}$ & $0,0089^{* * *}$ & $-0,1038^{* * *}$ \\
\hline & Desocupado & $0,0044^{* * *}$ & $0,1125^{* * *}$ & $-0,0082^{* * *}$ & $-0,1087^{* * *}$ \\
\hline
\end{tabular}




\begin{tabular}{|c|c|c|c|c|c|}
\hline & Variables & $\begin{array}{l}\text { Sin } \\
\text { estudios }\end{array}$ & Primaria & Media & Superior \\
\hline \multirow{2}{*}{$\begin{array}{l}\text { Actividad de la } \\
\text { madre }\end{array}$} & Inactivo & $0,0006^{* * *}$ & $0,0242^{* * *}$ & $0,0024^{* * *}$ & $-0,0273^{* * *}$ \\
\hline & Desocupado & $0,0019^{* * *}$ & $0,0583^{* * *}$ & 0,0005 & $-0,0608^{* * *}$ \\
\hline \multirow{3}{*}{$\begin{array}{l}\text { Tamaño del hogar } \\
\text { (miembros) }\end{array}$} & $>4$ & $-0,0032^{* * *}$ & $-0,1387^{* * *}$ & $-0,0337^{* * *}$ & $0,1757^{* * *}$ \\
\hline & 3 & $-0,0021^{* * *}$ & $-0,0968^{* * *}$ & $-0,0251^{* * *}$ & $0,1242^{* * *}$ \\
\hline & 2 & $-0,0011^{* * *}$ & $-0,0475^{* * *}$ & $-0,0081^{* * *}$ & $0,0569^{* * *}$ \\
\hline \multirow{3}{*}{$\begin{array}{l}N^{\circ} \text { de hermanos } \\
16 \text { años }\end{array}$} & 1 & $0,0108^{* * *}$ & $0,2507^{* * *}$ & $-0,0138^{* * *}$ & $-0,2477^{* * *}$ \\
\hline & 2 & $0,0294^{* * *}$ & $0,3931^{* * *}$ & $-0,0848^{* * *}$ & $-0,3377^{* * *}$ \\
\hline & $>3$ & $0,0644^{* * *}$ & $0,5000^{* * *}$ & $-0,1665^{* * *}$ & $-0,3988^{* * *}$ \\
\hline \multirow{2}{*}{ Mercado laboral } & T.D. juvenil & $-0,0012$ & -0.0445 & $-0,0051$ & 0,0509 \\
\hline & T.I. juvenil & $-0,0071^{* * *}$ & $-0,2673^{* * *}$ & $-0,0311^{* * *}$ & $0,3056^{* * *}$ \\
\hline Oferta & \# estudiantes IES/\# IES & $-0,0000^{* * *}$ & $-0,0000^{* * *}$ & $-0,0000^{* * *}$ & $0,0000^{* * *}$ \\
\hline \multirow{2}{*}{ Ingreso del hogar } & $1 £ S M L V £ 2$ & $-0,0006^{* * *}$ & $-0,0234^{* * *}$ & $-0,0032^{* * *}$ & $0,0273^{* * *}$ \\
\hline & $\mathrm{SMLV}>2$ & $-0,0019^{* * *}$ & $-0,0797^{* * *}$ & $-0,0153^{* * *}$ & $0,0970^{* * *}$ \\
\hline Ciudad & Bogotá & $-0,0005^{* * *}$ & $-0,0213^{* * *}$ & $-0,0033^{* * *}$ & $0,0251^{* * *}$ \\
\hline \multirow{6}{*}{ Cohortes } & $\mathrm{C} 2$ & $-0,0012^{* * *}$ & $-0,0542^{* * *}$ & $-0,0122^{* * *}$ & $0,0676^{* * *}$ \\
\hline & $\mathrm{C} 3$ & $-0,0011^{* * *}$ & $-0,0514^{* * *}$ & $-0,0111^{* * *}$ & $0,0637^{* * *}$ \\
\hline & $\mathrm{C} 4$ & $-0,0017^{* * *}$ & $-0,0801^{* * *}$ & $-0,0224^{* * *}$ & $0,1044^{* * *}$ \\
\hline & C5 & $-0,0019^{* * *}$ & $-0,0986^{* * *}$ & $-0,0324^{* * *}$ & $0,1330^{* * *}$ \\
\hline & C6 & $-0,0019 * * *$ & $-0,0953^{* * *}$ & $-0,0306^{* * *}$ & $0,1278^{* * *}$ \\
\hline & $\mathrm{C} 7$ & $-0,0020^{* * *}$ & $-0,0869^{* * *}$ & $-0,0189^{* * *}$ & $0,1079 * * *$ \\
\hline \multirow{4}{*}{ Año } & 2012 & $-0,0007^{* * *}$ & $-0,0307^{* * *}$ & $-0,0043^{* * *}$ & $0,0359^{* * *}$ \\
\hline & 2011 & $-0,0002^{* * *}$ & $-0,0093^{* * *}$ & $-0,0012^{* * *}$ & $0,0107^{* * *}$ \\
\hline & 2010 & $-0,0000$ & $-0,0006$ & $-0,0000^{* * *}$ & 0,0007 \\
\hline & 2009 & $0,0002^{* * *}$ & $0,0090^{* * *}$ & $0,0009^{* * *}$ & $-0,0102^{* * *}$ \\
\hline \multicolumn{2}{|c|}{ Pseudo Log-likelihood } & & -152319 & & \\
\hline \multicolumn{2}{|l|}{ Test Wald } & & 0,0000 & & \\
\hline \multicolumn{2}{|l|}{ Observaciones } & & 156370 & & \\
\hline
\end{tabular}

Fuente: Elaboración propia.

Nota: Significancia al: ${ }^{* * *} 0.01,{ }^{* *} 0.05,{ }^{*} 0.1$. Errores estándar robustos. Individuo de referencia: hombre, de padres sin estudios (o analfabeta), ocupados, en un hogar pequeño, sin hermanos, con un ingreso del hogar menor a 1 SMLV, nacido en la cohorte 1, que reside fuera de Bogotá, en el año 2008. 
La tabla 4 muestra los resultados de los efectos marginales de elegir educación superior por cohortes, condicionados a las características personales, familiares y del entorno del individuo. De esta manera, se resalta la variable género, cuyos coeficientes son significativos para los cuatro años. Se muestra que ser mujer incrementa la probabilidad de demandar educación superior en todas las cohortes.

Con respecto a las características de los padres, tener padre y madre con educación media y superior incrementa la probabilidad de demandar educación superior, además se puede apreciar que este efecto es mayor para las cohortes de mayor edad. Esto sugiere que, a medida que se avanza en el tiempo, las cohortes más jóvenes tendrán padres con niveles de educación más altos, por lo que estos resultados aportan a la idea de que individuos con familias más educadas o con tradiciones educativas alcanzarán mayores niveles de educación. En esta misma línea, tener padre inactivo o desempleado disminuye la probabilidad de demandar educación superior para todas las cohortes.

En lo que respecta a la madre, el ser desempleada o inactiva no parece influir en la demanda de educación de los hijos. Este resultado sugiere que la no participación de la madre en el mercado laboral no representa una gran barrera para los jóvenes que quieren acceder a mayores niveles educativos. Con respecto a la estructura del hogar, tener hermanos menores de 16 años disminuye la probabilidad de acceder a la educación superior, mientras el tamaño del hogar no parece afectar negativamente la probabilidad del acceso a este nivel.

El ingreso del hogar muestra que entre mayor sea este, entonces mayor es la magnitud de la probabilidad de demandar educación superior. Además, dicha probabilidad es mayor para las cohortes de mayor edad. Por otra parte, la variable Bogotá muestra que no existe una influencia significativa de la locación en la demanda de educación superior entre cohortes. Este resultado sugiere un avance en igualdad de oportunidades entre residir en cualquiera de las regiones del país y acceder a la educación superior.

Finalmente, el hecho de pertenecer a cualquiera de las cohortes y demandar educación superior entre los años 2009 al 2012 comparados con el 2008 tiene una probabilidad positiva, lo cual sugiere un panorama favorable en acceso a la educación superior en el tiempo.

Antes de analizar las probabilidades condicionales, debemos tener en cuenta que junto con los resultados descritos hasta ahora en esta sección, los efectos marginales por cohortes para cada año muestran que existen diferencias significativas en el tiempo y entre cohortes. 


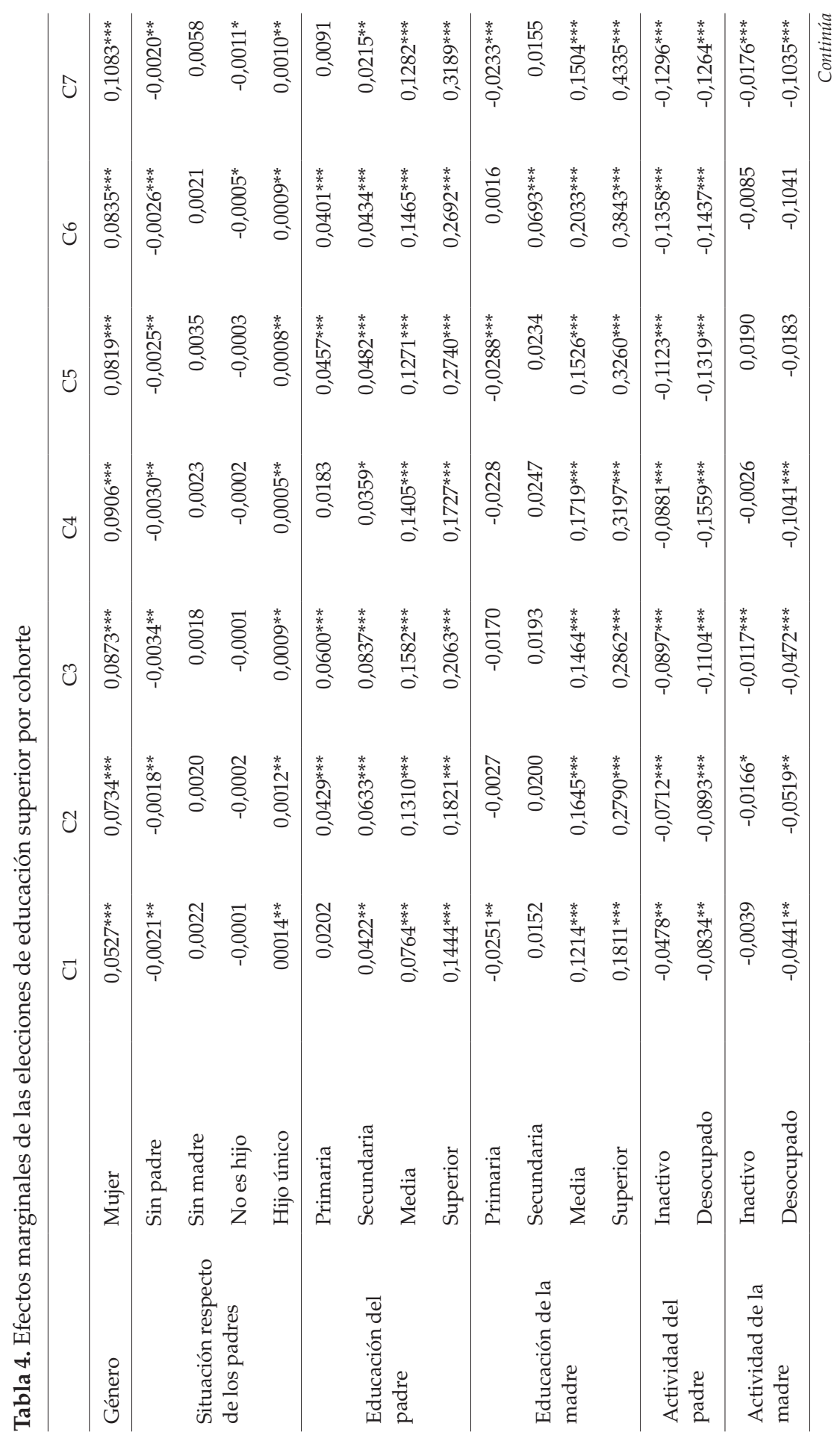




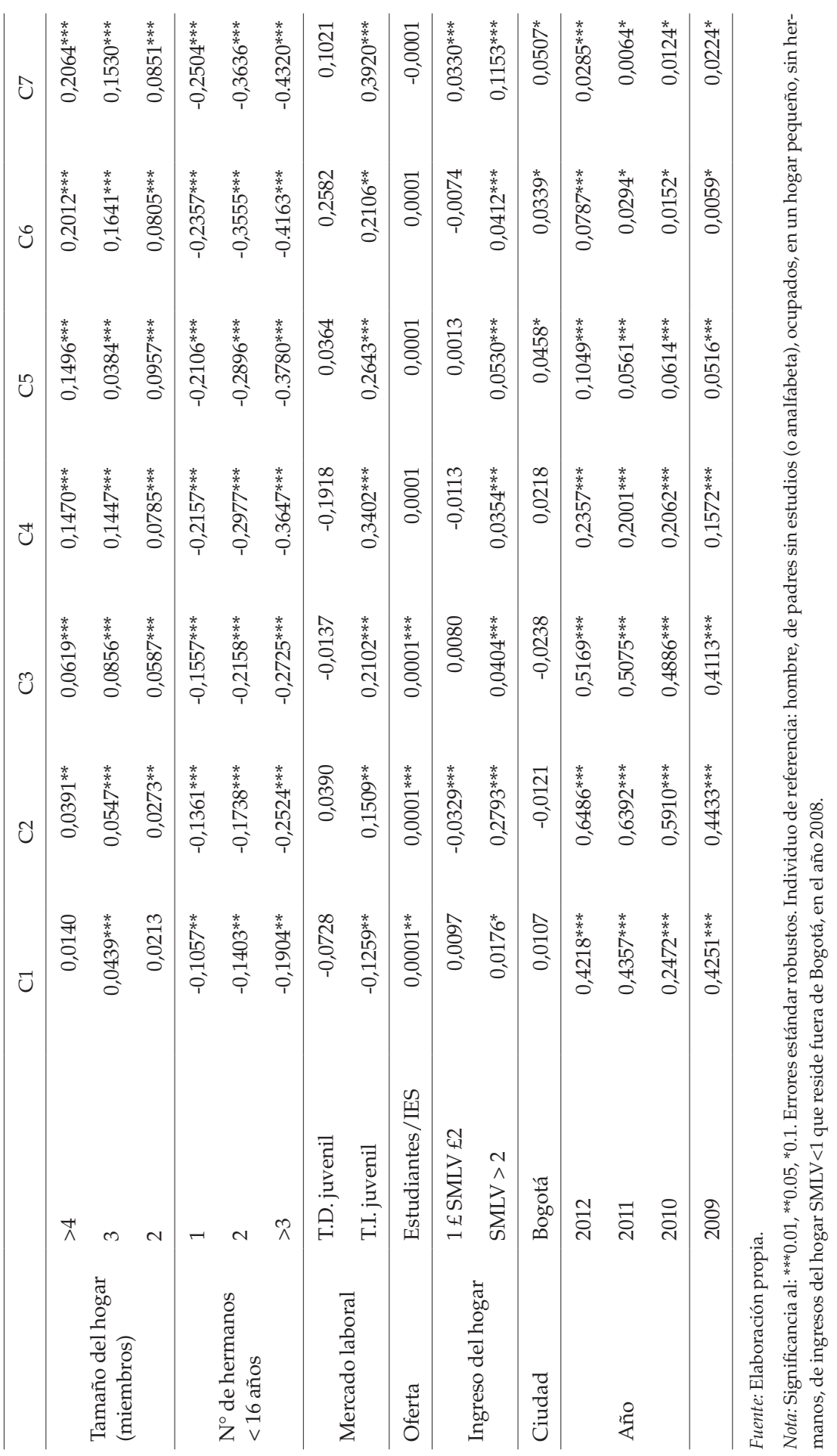


En el análisis de las predicciones de las probabilidades, en la figura 4, se puede observar un comportamiento creciente en la probabilidad de demandar mayores niveles educativos en el trascurso del periodo de análisis. De este modo, la probabilidad de tener un nivel de educación medio es la más alta en los cinco años, en 2008 la probabilidad es del $48 \%$ y en 2012 del $42 \%$. Este comportamiento en la probabilidad de acceder a mayores niveles de educación ha permitido que cada vez sea mayor la probabilidad de alcanzar los niveles de educación superior. Asimismo, el ritmo de crecimiento en la probabilidad de acceder a la educación superior está por encima del 30\% en el 2008 y apenas un $5 \%$ mayor en el 2012, pero su tendencia es creciente.

En cuanto a la probabilidad de quedarse sin estudios/analfabeta, esta es la menor, ubicándose por debajo del $5 \%$ en todos los años. Estos resultados pueden derivarse del esfuerzo del Ministerio de Educación por cerrar las brechas en inequidad por medio de una mayor participación de la sociedad en el sistema educativo, principalmente, por medio de proyectos de cobertura como Programa de alimentación escolar, Gratuidad educativa, Ni uno ni menos, Familias en acción, Proyecto Ícaro, y la gratuidad, entre otros.

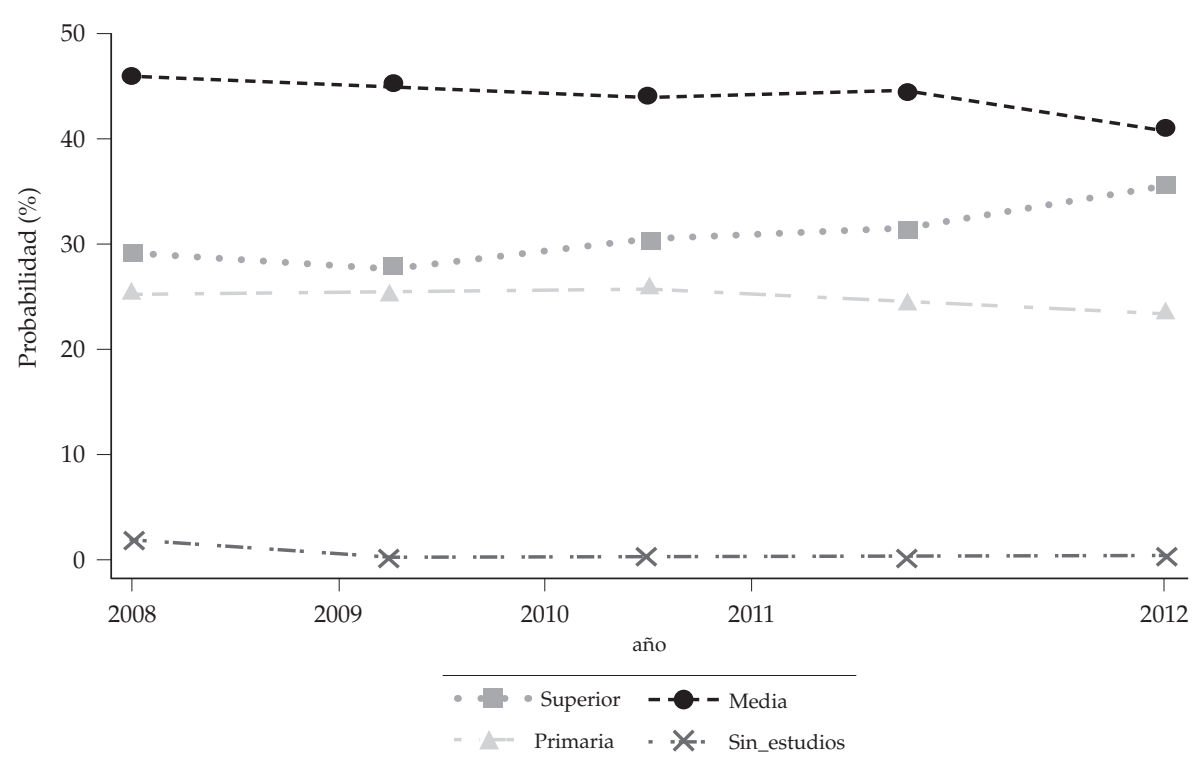

Figura 4. Probabilidad de participar en los niveles de educación (o sin educación) en Colombia

Fuente: Elaboración propia.

Nota: Individuo de referencia: hombre, de padres sin estudios (o analfabetas) cuya actividad laboral es estar ocupados, sin hermanos, en un hogar pequeño, de ingresos del hogar $\mathrm{SMLV}<1$ que reside por fuera de Bogotá D.C., en el año 2008. 
Si bien ha existido un avance en cada uno de los niveles obligatorios y posobligatorios, la pregunta sería dado que existen diferencias significativas en las características del individuo y su familia, en la probabilidad de acceder a mayores niveles educativos, ¿el aumento en la probabilidad ha sido igual para todas las personas en la sociedad? Esta pregunta es relevante, en el sentido de que lo que se busca no es solo incrementar la cobertura, sino que, al mismo tiempo, el acceso sea equitativo. Puesto que existen diferencias estadísticas, se analizan la predicción de las probabilidades condicionadas a las cohortes de nacimiento de los individuos.

Las figuras 5 y 6 muestran que la evolución no ha sido igual para todos los individuos en el tiempo de acuerdo con la cohorte de nacimiento. La figura 5 muestra, que los individuos que tienen mayores probabilidades de acceder a mayores niveles educativos son aquellos que pertenecen a las cohortes más jóvenes. En esta última cohorte, la probabilidad de participar en educación superior se mantuvo durante los cuatro años por encima del $35 \%$ y para la cohorte mayor la probabilidad era del 30\%. Sin embargo, la evolución de la probabilidad de los mayores aumentó y se cerró la brecha. Para el año 2012 las probabilidades de las dos cohortes en este nivel eran similares.

De igual forma, la figura 5 muestra un avance en la probabilidad de demandar mayores niveles de educación, al mantener la educación superior un comportamiento creciente. Es decir, en el tiempo los individuos más jóvenes cada vez tienen una mayor probabilidad de alcanzar mayores niveles educativos, en especial los niveles posobligatorios (media y superior).

En la figura 6 se observa la evolución en la participación en la educación superior para las cohortes mayor y menor. La participación de la cohorte 7 se ha mantenido alrededor de un $40 \%$ y su crecimiento entre los años 2008 y 2012 fue muy poco, alrededor de 2 puntos porcentuales. Sin embargo, en esta figura es importante destacar que en el año 2008 existía una brecha en la participación entre las dos cohortes. Esta brecha casi se cerró debido al crecimiento considerable de la participación de la cohorte 1 que pasó de un $10 \%$ a aproximadamente un $40 \%$. Esto pudo haber sido originado por las políticas de los dos últimos gobiernos que han impulsado la educación técnica y tecnológica con apoyo del SENA. 


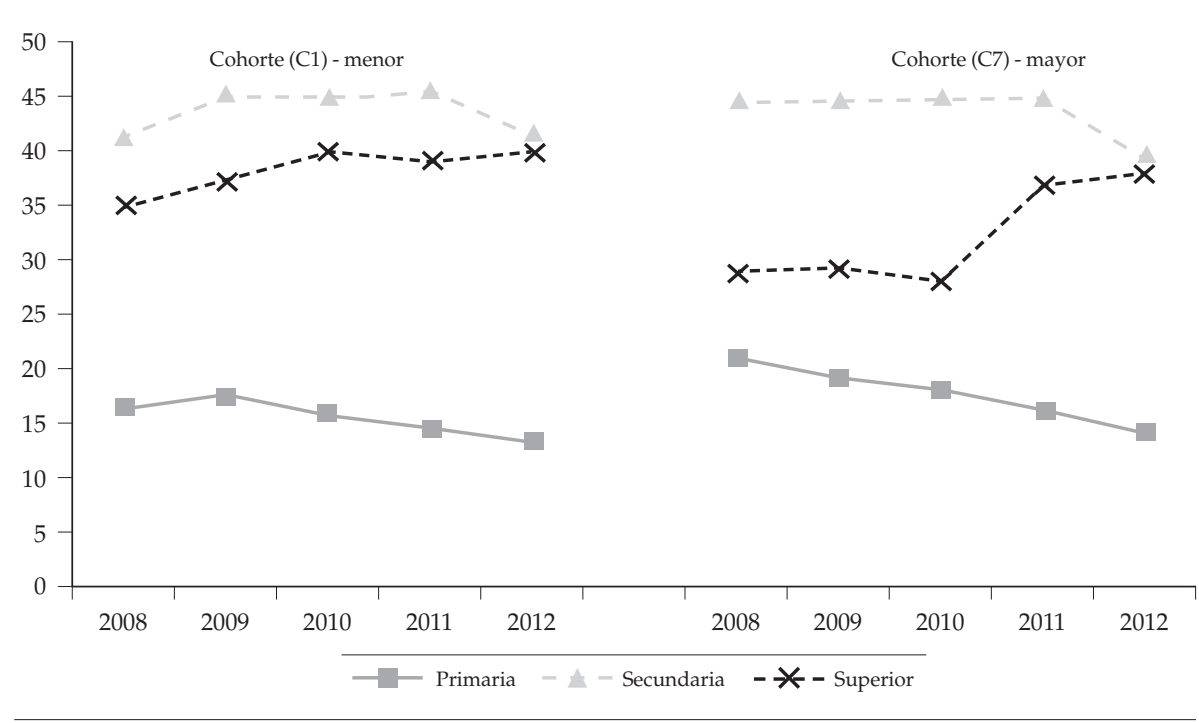

Figura 5. Probabilidad de participar en los niveles de educación según la cohorte Fuente: Elaboración propia con GEIH.

Nota: Individuo de referencia: hombre, de padres sin estudios (o analfabetas) cuya actividad laboral es estar ocupados, sin hermanos, en un hogar pequeño, con un ingreso del hogar inferior a 1 SMLV, que reside por fuera de Bogotá D.C., en el año 2008.

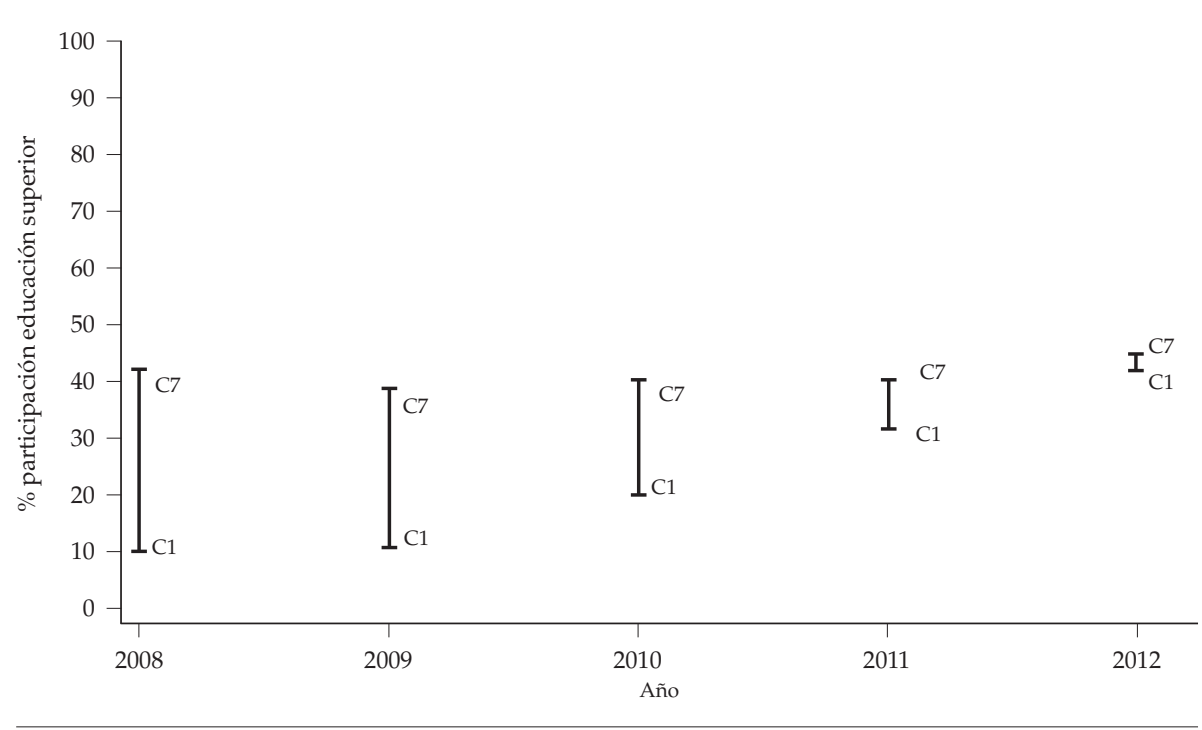

Figura 6. Evolución de la participación en educación superior para la cohorte mayor y menor

Fuente: Elaboración propia con GEIH.

Nota: C1 cohorte 1, C7 cohorte 7. 


\section{Conclusiones}

Este artículo provee un análisis de la relación entre las características familiares y las oportunidades de acceder a la educación posobligatoria (educación media y educación superior) en Colombia y su respectiva evolución entre cohortes de nacimiento. Los resultados indican que este tipo de elecciones educativas están condicionadas a características personales, familiares y del entorno más próximo del individuo. Este análisis confirma los resultados reportados sobre la expansión en el acceso a los diferentes niveles educativos, pero el acceso todavía es muy limitado a algunos grupos sociales.

Los resultados muestran que factores personales como el género (ser hombre), influye negativamente en la probabilidad de demandar mayores niveles de educación. En segundo lugar, la educación de los padres, variable relacionada con el ingreso familiar y el ambiente intelectual en el que se desarrolla el individuo (Rahona, 2006; Valiente, 2003) y como aproximación del grado de habilidad de los hijos (Leibowitz, 1974), aumenta la probabilidad de demandar mayores niveles de educación. En tercer lugar, la situación laboral, variable que refleja el estatus económico de la familia, muestra que tener padre ocupado influye positivamente en la probabilidad de demandar mayores niveles educativos (Albert, 1998). En cuarto lugar, el número de hermanos menores de dieciséis años, características que representan la estructura familiar y una aproximación del stock de inversión total que una familia realiza en cada hijo influyen negativamente en la demanda educativa (González, 2011). Por otra parte, el ingreso del hogar muestra que entre mayor sea este, mayor será la probabilidad de demandar niveles de educación superior.

Este artículo también confirma un leve aumento en la igualdad de oportunidades educativas, ya que cada vez son más las probabilidades de alcanzar mayores niveles de educación formal tanto en el tiempo como por cohortes. No obstante, frente a la pregunta si el avance ha sido equitativo, los resultados indican que en la educación obligatoria no existen diferencias entre las clases sociales, pero para los niveles posobligatorios los hogares con ingresos más altos tienen una mayor probabilidad de alcanzar la educación superior.

En suma, estos resultados sugieren la necesidad de una política que reduzca el impacto de las características familiares en el acceso a la educación media y superior. En este sentido, dado que los objetivos de cobertura para la educación básica (primaria y secundaria) parecen estarse cumpliendo, y que padres más educados incrementan la probabilidad de que sus hijos alcancen mayores niveles de educación, una alternativa es extender la educación media, especialmente el alcanzar el título de bachillerato al $100 \%$ de la población en edad escolar. Además, es necesario trabajar en el acceso equitativo de los hogares con ingresos más 
bajos hacia los niveles de educación superior (técnica, tecnológica y universitaria) con programas de becas completas con financiación público/privada que cubran los costos directos e indirectos y que permitan un acceso real de algunos grupos sociales menos favorecidos y que son mayoritarios en Colombia.

\section{Referencias}

Acevedo, S., Zuluaga, F. y Jaramillo, A. (2008). Determinantes de la Demanda por Educación Superior en Colombia. Revista de Economía del Rosario, 11(1), 121-148.

Ackemoglu, D. y Pischke, J. (2001). Changes in The Wage Structure, Family Income, and Children's Education. European Economic Review, 45(4), 890-904.

Albert, C. (1998). La Evolución de la Demanda de Enseñanza Superior en España. Hacienda Pública Española. Monografía Educación y Economía, 15(1) 119-137.

Albert, C. (2000). Higher Education Demand in Spain: The Influence of Labour Market Signals and Family Background. Higher Education, 40(2), 147-162.

Albert, C., Toharia, L. \& Davia, M. (2008). To Find or Not to Find a First "Significant" Job. Revista de Economia Aplicada, 16(1), 37-59.

Albert, C. y Davia, M. (2010). Education and Labour Market Transitions Amongst Compulsory Education Graduates and School Dropouts. Regional and Sectoral Economic Studies, Euro-American Association of Economic Development, 10(3), 5-22.

Albert V., C.; González C. G. y Mora J. J. "Determinantes de la demanda de educación universitaria en Colombia, 1980-2010", Revista de Economía Institucional 15, 29, 2013, pp. 169-194.

Barrera, F., Maldonado, D. y Rodríguez, C. (2012). Calidad de la Educación Básica y Media en Colombia: Diagnóstico y Propuestas. Documentos CEDE. Bogotá, Universidad de los Andes. Bogotá: Universidad de los Andes.

Becker, G. (1964). Human Capital Theoretical Analysis with Special Reference to Education. New York: Columbia University Press for NBER.

Brunello, G. y Checchi, D. (2007). Does School Tracking Affect Equality of Opportunity? New International Evidence. Economic Policy, 22(52), 781-861.

Caicedo, C. y Mora, J. J. (2013). ¿Igualdad salarial entre hombres y mujeres en Santiago de Cali? De la legislación a la realidad. Cali: Red de Observatorios Regionales del Mercado de Trabajo RED ORMET.

Cameron, S. y Heckman, J. (1998). Life Cycle Schooling and Dynamic Selection Bias: Models and Evidence for Five Cohorts of American Males. Journal of Political Economy, 106(2), 262-333. 
Cameron, S. y Heckman, J. (1999). The Dynamics of Educational Attainment for Blacks, Hispanics and Whites. NBER Working Paper 7249. National Bureau of Economic Research, Inc.

Cameron, A. y Trivedi, P. (2005). Microeconometrics: Methods and Applications. New York: Cambridge University Press.

Card, D. y Krueger, A. (1996). School Resources and Student Outcomes: An Overview of The Literature and New Evidence from North and South Carolina. Journal of Economic Perspectives, 10(4), 31-50.

Castillo, M. (2007). Desajuste Educativo por Regiones en Colombia: ¿Competencia por Salarios o por Puestos de Trabajo? Cuadernos de Economía, 26(46), 108-145.

Chevalier, A. y Lanot, G. (2002). The Relative Effect of Family Characteristics and Financial Situation on Educational Achievement. Education Economics, Taylor and Francis Journals, 10(2), 165-181.

Clancy, P. (1997). Higher Education in The Republic of Ireland: Participation and Performance. Higher Education Quarterly, 51(1), 86-106.

Clancy, P. (2001). College Entry in Focus: A Fourth National Survey of Access to Higher Education. Dublin: HEA.

Di Paolo, A. (2012). Parental Education and Family Characteristics: Educational Opportunities Across Cohorts in Italy and Spain. Revista de Economía Aplicada, 20(58), 119-146.

Ermisch, J. y Francesconi, M. (2001). Family Matters: impacts of Family Background on Educational Attainments. Economica, 68(270), 137-56.

Evans, W. y Schwab, R. (1995). Finishing High School and Starting College: Do Catholic Schools Make a Difference? Quarterly Journal of Economics, 110(4), 941-974.

Freeman, R. (1986). Demand for Education. En Ashenfelter, O. y Layard, R. Handbook of Labor Economics, North Holland: Amsterdam.

Fuller, W., Manski, C. y Wise, D. (1982). New Evidence on The Economic Determinants of Postsecondary Schooling Choices. The Journal of Human Resources, 17(4), 477-498.

Giannelli, G. y Monfardini, C. (2003). Joint Decisions on Household Membership and Human Capital Accumulation of Youths. The Role of Expected Earnings and Local Markets. Journal of Population Economics, 16(2), 265-286.

González, C. G., Giron, L. y Sanchez H. (2003). Algunos cálculos de la tasa de retorno de la educación para Cali, Revista Asuntos Económicos, 5(1), 7-25.

González, G. (2011). Análisis Económico de la Demanda de Educación Universitaria en Colombia: Un Análisis Cuantitativo. (Tesis doctoral, Universidad de Alcalá, España).

Greene, W. (2012). Econometrics Analysis. (7th ed.). New York: Prentice Hall. 
Gustman, A. y Steinmeier, T. (1981). The Impact of Wages and Unemployment on Youth Enrollment and Labor Supply. Review of Economics and Statistics, 63(4), 553-560.

Hanushek, E. (1992). The Trade-Off between Child Quantity and Quality, Journal of Political Economy, 100(1), 84-117.

Hanushek, E. y Wössmann, L. (2006). Does Educational Tracking Affect Performance and Inequality? Differences-in-Differences Evidence Across Countries. Economic Journal, 116(510), p. 63-76.

Hanushek, E. y Wössmann, L. (2008). The Role of Cognitive Skills in Economic Development. Journal of Economic Literature, 46(3), 607-68.

Haveman, R. y Wolfe, B. (1995). The Determinants of Children's Attainments: A Review of Method and Findings. Journal of Economic Literature, 33(4), 1829-1878.

Heller, D. (1997). Student Price Responses in Higher Education: An Update to Leslie and Brinkman. The Journal of Higher Education, 68(6), 624-659.

Kodde, D. y Ritzen, J. (1988). Direct and Indirect Effects of Parental Education Level on The Demand for Higher Education. The Journal of Human Resources, 23(3), 356-371.

Lauer, C. (2003). Family background, Cohort and Education: a French-German Comparison Based on a Multivariate Ordered Probit Model of Educational Attainment, Labour Economics, 10(2), 231-251.

Leibowitz, A. (1974). Home Investment in Children. Journal of Political Economy, 82(2), 111-131.

Leslie, L. y Brinkman, P. (1987). Student Price Response in Higher Education. Journal of Higher Education, 58(18), 1-204.

Light, A. (1995). Hazard Model Estimates of the Decision to Re-enroll in School. Labour Economics, 2(4), 381-406.

Lillard, L. y Robert, J. (1994). Intergenerational Educational Mobility: Effects of Family and State in Malaysia. Journal of Human Resources, 29(4), 1126-1166.

MEN (2012). Estadísticas de la Educación Superior. Bogotá: Ministerio de Educación Nacional de Colombia.

MEN (2013). Estadísticas de la Educación Superior. Bogotá: Ministerio de Educación Nacional de Colombia.

Mincer, J. (1974). Schooling, Experience and Earnings. New York: National Bureau of Economic Research.

Modrego, A. (1987). Demanda de Educación Superior. Resultados de la Estimación de un Modelo de Educación Superior para la Provincia de Vizcaya. Ekonomiaz, 19(1), 87-96.

Mora, J. J. (2008). Sobre-Educación en el Mercado Laboral Colombiano. Revista de Economía Institucional, 10(19), 293-309. 
Mora, J. y Muro, J. (2008). Sheepskin Effects by Cohorts in Colombia. International Journal of Manpower, 29(2), 111-121.

Neal, D. (1997). The Effects of Catholic Secondary Schooling on Educational Achievement. Journal of Labor Economics, 15(1), 98-123.

O’Brien, M. y Jones, D. (1999). Children, Parental Employment and Educational Attainment: An English Case Study. Journal of Economics, 23(5), 599-621.

Ordovensky, J. (1995). Effects of Institutional Attributes on Enrolment Choice: Implications for Postsecundary Vocational Education. Economics of Education Review, 14(4), 335-350.

Osterbeek, H. y Van Ophem, H. (2000). Schooling Choices: Preferences, Discount Rates, and Rates of Return. Empirical Economics, 25(1), 15-34.

Petrongolo, B. y San Segundo, M. (1998). Staying-on at School at Sixteen: The Impact of Labor Market Conditions in Spain. UC3M Working papers, 98-69. Madrid, Universidad Carlos III de Madrid.

Rahona, M. (2006). La Influencia del Entorno Socioeconómico en la Realización de Estudios Universitarios: Una Aproximación al Caso Español en la Década de los Noventa. Revista de Economía Pública, 178(3), 55-80.

Ramírez, A. (2008). Modelos de Elección Discreta: Una Aplicación a la Demanda por Cupos Universitarios en Colombia. Revista Universidad EAFIT, 44(152), 66-79.

Rice, P. (1987). The Demand for Post-Compulsory Education in the UK and the Effects of Educational Maintenance Allowances. Económica, 54(216), 465-475.

Rice, P. (1999). The Impact of Local Labour Markets on Investment in Further Education: Evidence from the England and Wales Cohort Studies. Journal of Population Economics, 12(2), 287-312.

San Segundo, M. (2003). Origen Socioeconómico y Capital Humano. En San Segundo, Calidad, Igualdad y Equidad en la Educación (pp. 1-35). Madrid: Biblioteca nueva.

Sánchez, F. y Núñez, J. (2003). A Dynamic Analysis of Human Capital, Female Work-Force Participation, Returns to Education and Changes in Household Structure in Urban Colombia, 1976 -1998. Colombian Economic Journal, 1(1), 1-149.

Sánchez, F. y Zúñiga, J. (2012). La Deserción en la Educación Superior en Colombia durante la Primera Década del Siglo XXI. ¿Por qué ha aumentado tanto? Documentos CEDE. Bogotá: Universidad de los Andes-CEDE.

Sanders, W. y Krautmann, A. (1995). Cotholic Schools, Dropout Rates, and Educational Attainment. Economic Inquiry, 33(2), 217-233. 
Tenjo, J. (2002). Demanda por Educación Superior: Proyecciones para los Siguientes Quince Años. Documentos de trabajo. Bogotá: Pontificia Universidad Javeriana.

Valiente, A. (2003). La demanda de Educación Universitaria y el Rendimiento Privado de la Educación en España. Valladolid: Secretariado de Publicaciones e Intercambio Editorial. Universidad de Valladolid.

Van Tilberg, E. y DuBois, J. (1989). Literacy Students Perceptions of Successful Participation in adult Education: A Cross-Cultural Approach Through Expectancy Valence. Proceedings of The 30th Annual Adult Education Research Conference, University of Wisconsin, Madison, WI.

Whelan, C. y Hannan, D. (1999). Class Inequalities in Educational Attainment Among the Adult Population in the Republic of Ireland. The Economic and Social Review, 30(3), 285-307.

Willis, R. y Rosen, S. (1979). Education and Self Selection. The Journal of Political Economy, 87(5), S7-S36. 
